\title{
Dream Teens: Dar voz a la juventud. El Impacto de un proyecto de participación social de los Jóvenes en Portugal
}

\author{
Cátia Santos Branquinho \\ Universidad de Lisboa, Portugal
}

\author{
Diego Gomez-Baya \\ Universidad de Huelva, España
}

\author{
Margarida Gaspar de Matos \\ Universidad de Lisboa, Portugal / Universidad Paul Sabatier, Francia
}

\section{RESUMEN}

Considerada un derecho fundamental la participación de los jóvenes sigue siendo poco priorizada. Con el objetivo de promover la voz, la participación social, y una ciudadanía más activa en los jóvenes portugueses, se creó la red Dream Teens. Tras dos años de proyecto, se realizaron un estudio cualitativo de su voz (estudio 1) y un estudio cuantitativo para examinar el impacto de esta red en los participantes (estudio 2). En el estudio 1, participaron un total de 108 jóvenes, con una media de 15,73 años $( \pm 1,80)$, mayoritariamente chicas $(71,30 \%)$. Después de categorizar los temas discutidos, se ha constatado una preferencia por cuestiones relacionadas con sus amigos y el pensamiento crítico respecto a la sociedad. En el estudio 2, participó una muestra de 46 jóvenes, con una edad media de 16,13 años $( \pm 1,89)$, también en gran parte chicas $(78,26 \%)$. Tras la evaluación pre y post-test, no se encontraron diferencias estadísticamente significativas, aunque las percepciones de los Dream Teens mostraron una mejoría, con una mayor participación en voluntariado y en actividades cívicas, y unas expectativas más positivas sobre el futuro. Así, aunque algunos resultados no fueron satisfactorios, las conclusiones de este estudio defienden la priorización de la voz de los jóvenes en temas relacionados con los amigos y la sociedad. También se ha constatado una percepción positiva en la mejora de sus competencias, junto al aumento de la participación en actividades de voluntariado y cívicos, además de de expectativas más positivas en relación al futuro.

Palabras clave

Juventud, participación social; impacto; análisis cualitativo; análisis cuantitativo.

\section{ABSTRACT}

Considered a fundamental right, the participation of young people continues to be a low priority. With the aim to promote the voice, social participation, and a more active citizenship of Portuguese youth, the Dream Teens network was created.

After two years of project, a qualitative study of their voice (study 1) and a quantitative study were carried out to examine the impact of this network on the participants (study 2). In study 1 , with a total of 108 young people involved, with an average of 15.73 years $( \pm 1.80)$, mostly girls $(71.30 \%)$, after a categorization of the topics discussed, a preference related to friends and critical thinking about society has been noted. In study 2, with a sample of 46 young people, with an average age of 16.13 years $( \pm 1.89)$, also in large part girls (78.26\%), after the pre and post-test application, no statistically significant differences were found, although the perceptions of the Dream Teens showed an improvement, with a greater participation in volunteering and civic activities, and more positive expectations about the future. Thus, although some results are incoherent, this study reveals the prioritization of the voice of young people in topics related to friends and society; of their positive perception in the improvement of their competences, together with the increase of participation in voluntary and civic activities, and of more positive expectations related to the future.

KeYWORDS

Youth social participation; impact; qualitative analysis; quantitative analysis. 
La participación social se define como un derecho fundamental (UNICEF, 2011) que pone de relieve el importante papel que tiene la juventud en su propio proceso de desarrollo personal, para el logro de competencias para la vida diaria, el conocimiento de los derechos humanos y una mayor participación ciudadana (United Nations, 2010). Varios autores han explorado varias formas de participación. $L a$ Escalera de la Participación de Hart (1992), los Niveles de Participación de Treseder (1997) o los Caminos para la Participación de Shier (2001), son algunos ejemplos han servido de apoyo para el desarrollo de programas ampliamente reconocidos de investigación participativa en los jóvenes, como los programas Youth Participatory Action Research (YPAR; Ozer \& Douglas, 2013) o Youth-Adult Partnership (Y-AP; Zeldin, Gauley, Krauss, Kornbluh, \& Collura, 2015). Estos dos programas han servido de base para el desarrollo de la metodología del proyecto Dream Teens (DT) que se presenta en este trabajo.

DT es un proyecto pionero en Portugal de priorización de la implicación, de las ideas y de la voz de los jóvenes en comportamientos relacionados con la salud y el bienestar, alentando una participación más activa y promoviendo la autonomía de la juventud en el liderazgo de medidas de investigación-acción (Branquinho, Matos, \& Equipa Aventura Social / Dream Teens, 2016; Matos et al., 2015). Este proyecto está fundamentado en los tres grandes principios de eficacia de los programas de participación juvenil: 1) la promoción de oportunidades de liderazgo; 2) el desarrollo de competencias para la vida; y 3) el apoyo de mentores adultos en el desarrollo juvenil (Frasquilho, et al., 2016; Lerner \& Lerner, 2013). Además, se integran los seis componentes propuestos por la Organización Mundial de la Salud (World Health Organization, 2014) que permiten: 1) identificar necesidades y establecer prioridades; 2) presentar un modelo facilitador de la planificación de acciones; 3) identificar estructuras y procesos de apoyo; 4) facilitar la evaluación y las implicaciones del proyecto; 5) garantizar la supervisión, la evaluación y la diseminación; y 6) promover el empoderamiento de sus participantes. En este proyecto, el desarrollo del conocimiento se relaciona directamente con la propia participación de los jóvenes, asumiendo las distintas formas de participación predominantes. Este trabajo de investigación presenta el impacto del desarrollo de un programa de investigación participativa con jóvenes.

\section{Metodología}

\section{Participantes}

La red DT estuvo formada por 147 jóvenes. La muestra final estuvo compuesta para el estudio cualitativo de la voz (estudio 1) por 108 jóvenes, ya que 39 jóvenes no contestaron a los desafíos lanzados, con una edad media de $15,73$ años ( $\pm 1,80)$, de género mayoritariamente femenino $(71,30 \%)$ y escolarizadas en Educación Secundaria (52,77\%). Para el estudio cuantitativo del impacto del proyecto DT (estudio 2), participaron un total de 46 jóvenes del I y II Encuentro Nacional de DT. Esta muestra presentó una edad media de 16,13 años ( \pm $1,89)$, compuesta mayoritariamente por chicas (el 78,26\%), y por estudiantes de Educación Secundaria $(65,22 \%)$. 


\section{Procedimiento}

El Proyecto DT fue enviado inicialmente para su aprobación al Centro Académico de la Facultad de Medicina de la Universidad de Lisboa (Portugal). El proyecto DT se inició en 2014 y contó con tres grandes fases. En la primera fase (año 1), con la intención de promocionar el proyecto entre los jóvenes portugueses de 11 a 18 años de edad, se establecieron diversas colaboraciones con instituciones dedicadas al trabajo con jóvenes, además de contactar con personalidades públicas de la televisión, el deporte, la música y el humor, para que dieran su imagen para anunciar el proyecto en las sociedades colaboradoras, en las redes sociales y en un canal de televisión nacional. En total, se reunieron 298 solicitudes, que fueron analizadas para examinar el cumplimiento de los criterios de inclusión, en concreto: una edad comprendida entre los 11 y los 18 años; la presentación de autorización parental; y la presentación de una carta de motivación. Después de eliminar las candidaturas que no cumplieron los requisitos y aquellas que estaban duplicadas, 147 pasaron a la fase siguiente, que consistió en una entrevista a través de Skype.

Integrados en la red Dream Teens, los jóvenes fueron dinamizados a través de diversos desafíos, teniendo como base los siguientes temas: 1) recursos personales y bienestar; 2) capital social; 3) amor y sexualidad; 4) consumo de sustancias y accidentalidad; 5) estilos de vida; y 6) ciudadanía y participación social. Los desafíos basados en estos seis temas sirvieron para promover la discusión activa de los DT y recoger sus voces. En esta tarea de discusión de desafíos, hasta 108 jóvenes colaboraron ac- tivamente. Paralelamente a este trabajo, después de haber promovido sus competencias y técnicas de investigación, y bajo la orientación de los investigadores sénior, los DT pusieron en marcha pequeños proyectos de investigación en sus contextos de vida. Durante este primer año, los DT tuvieron la oportunidad de elaborar una carta de 30 recomendaciones en el área de la salud, entregada al representante del Sr. Secretario de Estado en materia de Salud en el I Encuentro Nacional de DT. Durante este encuentro, los jóvenes más activos ( $n=$ 46) se conocieron por primera vez presencialmente, y se realizó la evaluación pre-test de este proyecto.

Terminada la primera fase, comenzó el año 2 del proyecto. La voz de los jóvenes fue integrada dentro del libro Adolescentes: navegação segura por águas desconhecidas, Lisboa: Coisas de Ler (publicado en portugués, inglés y francés), titulado en castellano Adolescentes: navegación segura por aguas desconocidas (Matos, 2015). Además, se llevaron a cabo varias presentaciones del proyecto DT y del estudio Health Behavior in School-aged Children Study (HBSC), también desarrollado en Portugal por el equipo Aventura Social, en las escuelas y autoridades de sus áreas de residencia. Además, se siguieron desarrollando proyectos individuales de investigación-acción por parte de los DT. El final de este segundo año estuvo marcado por el II Encuentro Nacional de Dream Teens, momento en el que se realizó la evaluación post-test del estudio, y en el que se realizó la presentación pública del libro y de los proyectos individuales de los jóvenes. 
Variables del estudio y diseño

\section{de análisis de Datos}

\section{Estudio 1}

Se llevó a cabo el estudio cualitativo de la voz de los jóvenes con una perspectiva exploratoria y descriptiva, de los comentarios ofrecidos a los desafíos lanzados. Con el apoyo del software Nvivo 10, fueron interpretados y clasificados en categorías y subcategorías tras un análisis temático (Branquinho, Cruz, \& Matos, 2017), tomando como base el modelo de KiaKeating, Dowdy, Morgan y Noam (2011) del desarrollo saludable de los jóvenes (ver figura 1).

\section{Estudio 2}

Se realizó un análisis cuantitativo con el programa estadístico SPSS versión 24, del impacto de esta red DT en los participantes, a nivel de sus perspectivas de futuro, su compromiso con actividades de voluntariado y de liderazgo en la comunidad, y en las dimensiones siguientes: sentimientos y competencias para la acción, competencias para la resolución de problemas, competencias interpersonales, humanitarismo, y sentimientos relacionados con la vida. Se llevó a cabo un análisis factorial exploratorio de todos los ítems incluidos en el cuestionario (medidos con una escala tipo Likert de 5 puntos, desde 1 = totalmente en desacuerdo a 5 = totalmente de acuerdo), que fueron extraídos de los siguientes instrumentos:

- Research and Action Self-efficacy (Ozer \& Schotland, 2011).

- Students' Life Satisfaction Scale (Huebner, 1991).

- Measuring Self-Efficacy in Youths (Muris, 2001).

- Active and Engaged Citizenship (Zaff, Boyd, Li, Lerner, \& Lerner, 2010).

- Profiles of Student Life: Attitudes and Behaviors (Search Institute, 2011).

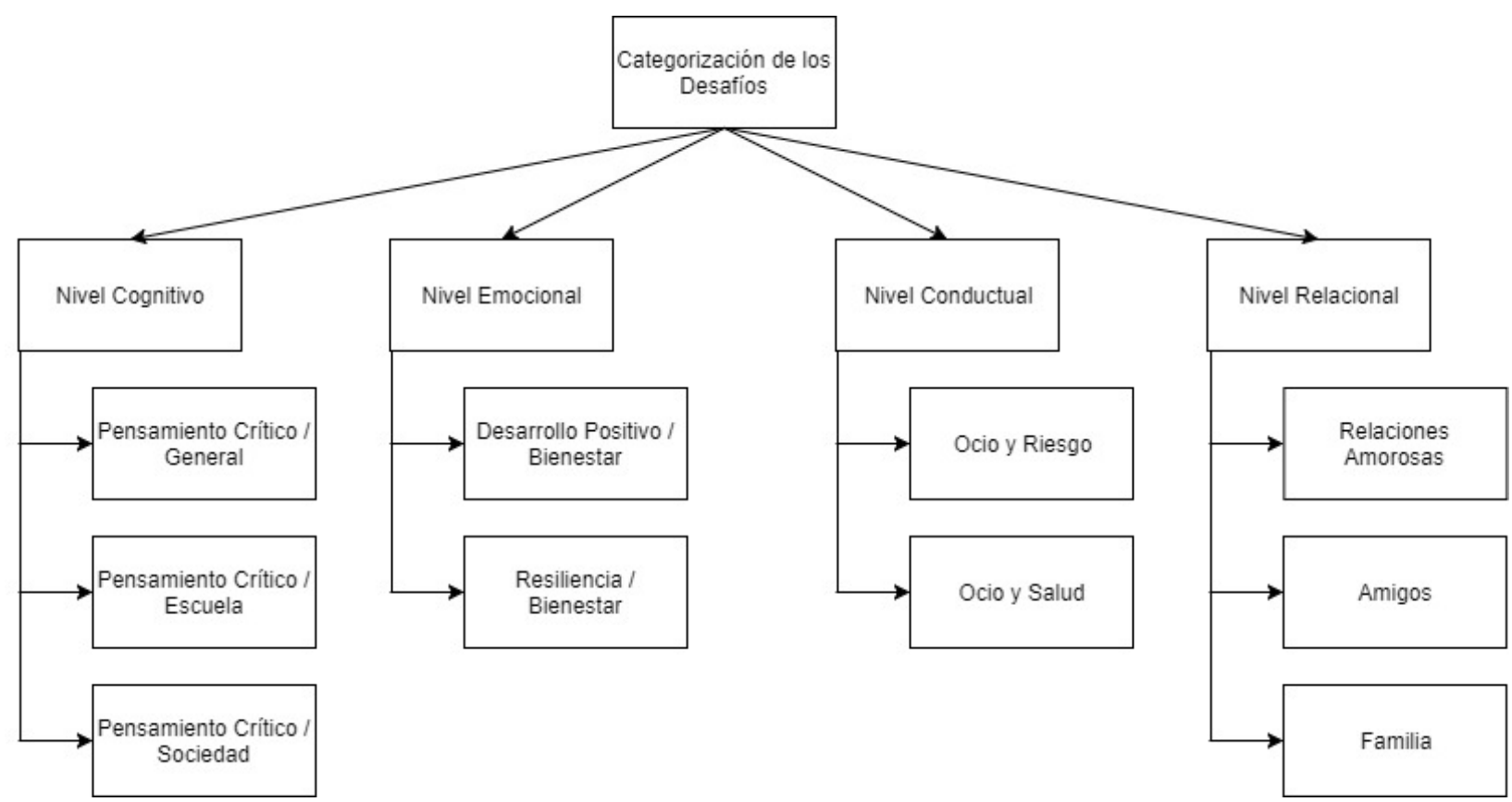

Figura 1. Clasificación de los desafíos.

Nota: Figura adaptada de Branquinho et al. (2017). 
Además, se estudiaron los estadísticos descriptivos de las variables de estudio. Para llevar a cabo el análisis de la comparación entre el pre-test y el post-test, se realizó la prueba $t$ de Student de muestras relacionadas.

\section{Resultados}

En el estudio 1 (Branquinho et al., 2017) relativo al análisis de los desafíos, se puede verificar que a pesar de que el número de desafíos lanzados se reparten en diversas categorías y subcategorías, los desafíos del nivel cognitivo en la subcategoría de pensamiento crítico relacionado con la sociedad y del nivel relacional en la subcategoría de amigos, presentaron un número promedio de respuestas superiores a los demás. Por el contrario, presentó un escaso número de respuestas la subcategoría de pensamiento crítico relacionado con la escuela, del nivel cognitivo (ver Tabla 1). En el estudio 2 (Branquinho \& Matos, 2018), se llevó a cabo un análisis factorial de los ítems del instrumento y se obtuvo un modelo con cinco factores: 1) sentimientos y competencias para la acción $(a=0,91), 2)$ competencias interpersonales $(a=0,75), 3)$ competencias para la resolución de problemas $(a=0,82), 4)$ humanitarismo ( $a$ $=0,83)$, y 5) sentimientos relacionados con la vida $(a=0,64)$. Esta estructura factorial alcanzó una varianza total explicada del $66,72 \%$. A continuación, se examinaron la percepción relativa al impacto del proyecto y la evaluación pre-post test de su participación en actividades de liderazgo y voluntariado, además de sus expectativas para el futuro.

Respecto a las percepciones del impacto del proyecto, se manifiesta una mejoría tras el año 1 en los indicadores relacionados con los sen-

Tabla 1

Categorías, subcategorías, número de desafíos, respuestas y promedio de respuestas para cada desafío

\begin{tabular}{|c|c|c|c|c|c|c|}
\hline \multirow[t]{2}{*}{ Categorías } & \multirow[t]{2}{*}{ Subcategorías } & \multicolumn{2}{|c|}{$\begin{array}{c}\text { Número de } \\
\text { desafíos }\end{array}$} & \multicolumn{2}{|c|}{$\begin{array}{c}\text { Respuestas } \\
\text { Desafíos }\end{array}$} & \multirow{2}{*}{$\begin{array}{c}\text { Promedio } \\
\text { Respuestas } \\
\text { M }\end{array}$} \\
\hline & & $\mathbf{n}$ & $\%$ & $\mathbf{n}$ & $\%$ & \\
\hline \multirow{4}{*}{ Nivel cognitivo } & Pensamiento Crítico / General & 22 & 19,30 & 195 & 15,35 & 8,86 \\
\hline & Pensamiento Crítico / Escuela & 5 & 4,39 & 29 & 2,28 & 5,80 \\
\hline & Pensamiento Crítico / Sociedad & 15 & 13,16 & 215 & 16,93 & 14,33 \\
\hline & Total & 42 & 36,84 & 439 & 34,57 & 10,45 \\
\hline \multirow{3}{*}{ Nivel emocional } & Desarrollo positivo / Bienestar & 21 & 18,42 & 215 & 16,93 & 10,24 \\
\hline & Resiliencia / Bienestar & 5 & 4,38 & 49 & 3,86 & 9,80 \\
\hline & Total & 26 & 22,81 & 264 & 20,79 & 10,15 \\
\hline \multirow{3}{*}{ Nivel conductual } & Ocio y Riesgo & 19 & 16,67 & 241 & 18,98 & 12,68 \\
\hline & Ocio y Salud & 10 & 8,77 & 102 & 8,03 & 10,20 \\
\hline & Total & 29 & 25,44 & 343 & 27,01 & 11,83 \\
\hline \multirow{5}{*}{ Nivel relacional } & Relaciones Amorosas & 9 & 7,89 & 109 & 8,58 & 12,11 \\
\hline & Amigos & 5 & 4,39 & 90 & 7,09 & 18,00 \\
\hline & Familia & 3 & 2,63 & 25 & 1,97 & 8,33 \\
\hline & Total & 17 & 14,91 & 224 & 17,64 & 13,18 \\
\hline & TOTAL & 114 & 100 & 1.270 & 100 & 11,1 \\
\hline
\end{tabular}

Nota:Tabla adaptada de Branquinho et al. (2017). 
timientos y competencias para la acción, las competencias interpersonales y las competencias para la resolución de problemas (ver Tabla 2), en concreto: «Los estudiantes como yo tene- mos el conocimiento y las competencias para participar en la toma de decisiones de nuestra escuela»; «Motivé a un grupo de jóvenes para trabajar en un asunto que nos preocupaba» y

\section{Tabla 2}

Percepciones de los Dream Teens - \% de jóvenes que informaron de mejoras percibidas después del proyecto $(\mathrm{N}=46)$

\begin{tabular}{|c|c|c|}
\hline \multirow{2}{*}{ Ítems } & \multirow{2}{*}{$\begin{array}{l}\text { Año } 1 \\
\text { Sí (\%) } \\
\end{array}$} & \multirow{2}{*}{$\begin{array}{l}\text { Año } 2 \\
\text { Sí (\%) } \\
\end{array}$} \\
\hline & & \\
\hline $\begin{array}{l}\text { 1. Si existen cuestiones que afecten a los estudiantes de mi escuela, haremos algo en } \\
\text { relación a eso. }\end{array}$ & & 78,26 \\
\hline $\begin{array}{l}\text { 2. Hablé en mi escuela con adultos sobre cuestiones que me gustaría mejorar en la } \\
\text { escuela. }{ }^{1}\end{array}$ & 63,04 & 65,22 \\
\hline $\begin{array}{l}\text { 3. Conseguí hacer una buena presentación ante los estudiantes de mi escuela sobre un } \\
\text { asunto que me preocupaba. }{ }^{1}\end{array}$ & 63,04 & 69,56 \\
\hline 4. Logré hacer una verdadera aportación en la mejora de mi escuela. ${ }^{1}$ & 65,22 & 65,22 \\
\hline 5. Es importante para mí contribuir a mi comunidad y a la sociedad. ${ }^{1}$ & 67,39 & 69,56 \\
\hline 6. Los profesores se implican realmente conmigo. ${ }^{1}$ & 63,04 & 56,52 \\
\hline 7. Creo que logré hacer una aportación a mi comunidad. ${ }^{1}$ & 65,22 & 73,91 \\
\hline $\begin{array}{l}\text { 8. Quiero tener una voz tan activa como sea posible en la toma de decisiones de mi } \\
\text { escuela. }\end{array}$ & 71,74 & 80,43 \\
\hline $\begin{array}{l}\text { 9. Los estudiantes como yo tenemos el conocimiento y las competencias para participar } \\
\text { en la toma de decisiones de nuestra escuela. }\end{array}$ & 73,91 & 67,39 \\
\hline 10. Conseguí colaborar en armonía con mis amigos. ${ }^{2}$ & 80,43 & 82,61 \\
\hline $\begin{array}{l}\text { 11. Si no tengo éxito en hacer valer mi opinión sobre un asunto, aprendo lecciones que } \\
\text { puedo usar la próxima vez. }{ }^{2}\end{array}$ & 73,91 & 73,91 \\
\hline 12. Consigo frecuentemente organizar a las personas para hacer las cosas. ${ }^{2}$ & 97,83 & 73,91 \\
\hline 13. Motivé a un grupo de jóvenes para trabajar en un asunto que nos preocupaba. ${ }^{2}$ & 73,91 & 71,74 \\
\hline 14. Conseguí conversar con una persona que apenas conocía. ${ }^{2}$ & 73,91 & 84,78 \\
\hline $\begin{array}{l}\text { 15. Si quiero mejorar la resolución de un problema en mi escuela, sé con qué adultos } \\
\text { debo hablar. }{ }^{2}\end{array}$ & 67,39 & 78,26 \\
\hline $\begin{array}{l}\text { 16. Si existen cuestiones que afectan a los jóvenes de mi ciudad o pueblo, haremos algo } \\
\text { en relación a eso. }{ }^{3}\end{array}$ & 73,91 & 65,22 \\
\hline $\begin{array}{l}\text { 17. Si quiero solucionar un problema de mi ciudad o pueblo, consigo trabajar de forma } \\
\text { efectiva con otros estudiantes en ese asunto. }{ }^{3}\end{array}$ & 69,56 & 71,74 \\
\hline $\begin{array}{l}\text { 18. Si quiero solucionar un problema de mi ciudad o pueblo, sé con qué adultos debo } \\
\text { hablar. }{ }^{3}\end{array}$ & 63,04 & 71,74 \\
\hline 19. Soy la clase de persona que realmente contribuye a su ciudad o pueblo. ${ }^{3}$ & 56,52 & 58,69 \\
\hline 20. Ayudo para reducir el hambre y la pobreza en el mundo. ${ }^{4}$ & 65,22 & 58,69 \\
\hline 21. Ayudo para hacer del mundo un lugar mejor en el que vivir. ${ }^{4}$ & 69,56 & 71,74 \\
\hline 22. Ayudo para asegurarme de que todas las personas son tratadas de forma justa. ${ }^{4}$ & 67,39 & 69,56 \\
\hline 23. Mi vida está bien. ${ }^{5}$ & 67,39 & 76,09 \\
\hline 24. Tengo lo que quiero en la vida. ${ }^{5}$ & 63,04 & 69,56 \\
\hline 25. Mi vida es mejor que la de la mayoría de los jóvenes de mi edad. ${ }^{5}$ & 65,22 & 67,39 \\
\hline
\end{tabular}

Notas: ' Factor 1. Sentimientos y competencias para la acción; ${ }^{2}$ Factor 2 . Competencias interpersonales; ${ }^{3}$ Factor 3. Competencias para la resolución de problemas; ${ }^{4}$ Factor 4. Humanitarismo; ${ }^{5}$ Factor 5 . Sentimientos relacionados con la vida. Tabla adaptada de Branquinho y Matos (2018). 
«Si existen cuestiones que afecten a los jóvenes de mi ciudad o pueblo, haremos algo en relación a eso». Al final del año 2 , se encontró una mejoría en los indicadores relacionados con los sentimientos y competencias para la acción, las competencias para la resolución de problemas y los sentimientos relacionados con la vida, como: «Si existen cuestiones que afecten a los estudiantes de mi escuela, haremos algo en relación a eso», "Quiero tener una voz tan activa como sea posible en la toma de decisiones de mi escuela», "Si quiero mejorar la resolución de un problema en mi escuela, sé con qué adultos debo hablar», y «Mi vida está bien». Además, se ha encontrado una percepción de evolución de las competencias interpersonales en ambos años del estudio, destacándose los indicadores siguientes: «Conseguí colaborar en armonía con mis amigos», «Si no tengo éxito en hacer valer mi opinión sobre un asunto, aprendo lecciones que puedo usar la próxima vez», «Consigo frecuentemente orga- nizar a las personas para hacer las cosas», y por último, «Conseguí conversar con una persona que apenas conocía».

En la evaluación pre-post test del impacto del proyecto, aplicando la prueba $t$ de Student para muestras relacionadas, no se encontraron cambios destacables en ninguna de las cinco dimensiones estudiadas (ver Tabla 3). Ninguna de las diferencias resultó significativa al nivel $p<0,01$.

Sin embargo, se evidencia una mayor participación en actividades relacionadas con las asociaciones de estudiantes (año $1=17,39 \%$ y año $2=31,82 \%$ ) y con la iglesia (año $1=19,56 \%$ y año $2=21,73 \%$ ). La participación en actividades de voluntariado al final del año 1 (95,30\%) y del año $2(90,91 \%)$ es masiva, y aunque presente una disminución tras el seguimiento, la integración en actividades de tutoría (año $1=$ $14,81 \%$ y año $2=18,18 \%$ ) y de apoyo de mentores adultos (año $1=0,00 \%$ y año $2=9,09 \%$ ) mostró un aumento sustancial (ver Tabla 4).

\section{Tabla 3}

Resultados del pre y post-test sobre el impacto del proyecto Dream Teens $(N=46)$

\begin{tabular}{|c|c|c|c|c|c|c|}
\hline \multirow{3}{*}{$\begin{array}{l}\text { Sentimientos y competencias } \\
\text { para la acción }\end{array}$} & \multicolumn{2}{|c|}{ Pre $(\mathrm{N}=39)$} & \multicolumn{2}{|c|}{ Post $(\mathrm{N}=39)$} & \multirow[b]{2}{*}{$t$} & \multirow[b]{2}{*}{$p$ bilatera } \\
\hline & $M$ & $D T$ & $M$ & $D T$ & & \\
\hline & 3,44 & 83 & 3,32 & ,76 & ,78 & ,441 \\
\hline \multirow{3}{*}{ Competencias interpersonales } & \multicolumn{2}{|c|}{ Pre $(\mathrm{N}=43)$} & \multicolumn{2}{|c|}{ Post $(\mathrm{N}=43)$} & & \\
\hline & $M$ & $D T$ & $M$ & $D T$ & $t$ & $p$ \\
\hline & 3,81 & ,75 & 3,53 & ,73 & 2,10 & ,042 \\
\hline \multirow{3}{*}{$\begin{array}{l}\text { Competencias para la } \\
\text { resolución de problemas }\end{array}$} & \multicolumn{2}{|c|}{ Pre $(\mathrm{N}=46)$} & \multicolumn{2}{|c|}{ Post $(N=46)$} & & \\
\hline & $M$ & $D T$ & $M$ & $D T$ & $t$ & $p$ \\
\hline & 3,08 & 79 & 2,88 & 82 & 1,69 & 097 \\
\hline \multirow{3}{*}{ Humanitarismo } & \multicolumn{2}{|c|}{ Pre $(\mathrm{N}=46)$} & \multicolumn{2}{|c|}{ Post $(\mathrm{N}=46)$} & & \\
\hline & $M$ & $D T$ & $M$ & $D T$ & $t$ & $p$ \\
\hline & 4,46 & 69 & 4,46 & ,71 & ,33 & ,740 \\
\hline \multirow{3}{*}{$\begin{array}{l}\text { Sentimientos relacionados con } \\
\text { la vida }\end{array}$} & \multicolumn{2}{|c|}{ Pre $(\mathrm{N}=46)$} & \multicolumn{2}{|c|}{ Post $(\mathrm{N}=46)$} & & \\
\hline & $M$ & $D T$ & $M$ & $D T$ & $t$ & $p$ \\
\hline & 3,79 & 80 & 3,75 & 88 & 07 & 944 \\
\hline
\end{tabular}

Nota: Tabla extraída de Branquinho y Matos (2018). 
Tabla 4

Participación en actividades de liderazgo y perspectivas de futuro $(n=46)$

\begin{tabular}{|c|c|c|}
\hline \multirow{2}{*}{ Participación en actividades de Liderazgo } & \multirow{2}{*}{$\begin{array}{l}\text { Año } 1 \\
\text { Sí }(\%)\end{array}$} & \multirow{2}{*}{$\frac{\text { Año } 2}{\text { Sí (\%) }}$} \\
\hline & & \\
\hline Relacionadas con actividades en la comunidad & 52,17 & 52,27 \\
\hline Relacionadas con la iglesia & 19,56 & 21,73 \\
\hline Relacionadas con las asociaciones de estudiantes & 17,39 & 31,82 \\
\hline Relacionadas con un partido político & 8,69 & 9,09 \\
\hline Relacionadas con un equipo & 30,43 & 15,91 \\
\hline Relacionadas con una banda & 4,35 & 9,09 \\
\hline Otras actividades de liderazgo & 21,74 & 18,18 \\
\hline Participación en actividades de voluntariado & 96,30 & 90,91 \\
\hline Participación en actividades de apoyo de mentores adultos & 0,00 & 9,09 \\
\hline Participación en actividades de tutoría & 14,81 & 18,18 \\
\hline \multirow{2}{*}{$\begin{array}{l}\text { Perspectivas de futuro } \\
\text { ¿Qué esperas hacer cuando acabes la educación Secundaria? }\end{array}$} & Año 1 & Año 2 \\
\hline & Sí (\%) & Sí (\%) \\
\hline Seguir los estudios en una universidad o institución educativa superior & 91,30 & 80,43 \\
\hline Conseguir un trabajo & 10,87 & 21,74 \\
\hline Buscar un trabajo & 6,52 & 19,56 \\
\hline Seguir los estudios haciendo un curso de formación técnica o profesional & 2,17 & 2,17 \\
\hline Cualificación Profesional & 4,35 & 2,17 \\
\hline Estar desempleado & 0,00 & 0,00 \\
\hline No lo sé & 4,35 & 2,17 \\
\hline ¿Qué esperas hacer dentro de 10 años? & Sí (\%) & Sí (\%) \\
\hline Trabajar en mi área de interés & 73,91 & 71,74 \\
\hline Estar en la universidad & 8,70 & 8,70 \\
\hline Formar una familia y trabajar en mi área de interés & 6,52 & 4,35 \\
\hline Estar viviendo experiencias por el mundo & 2,17 & 0,00 \\
\hline Estar haciendo algo importante para la sociedad & 0,00 & 2,17 \\
\hline Trabajar en una empresa multinacional & 0,00 & 2.17 \\
\hline Formar una familia & 0,00 & 2,17 \\
\hline No lo sé & 8,70 & 8,70 \\
\hline
\end{tabular}

Nota: Tabla extraída de Branquinho y Matos (2018).

Finalmente, en relación con las perspectivas de futuro (ver Tabla 4), la mayoría de los jóvenes piensa en continuar con los estudios en una universidad o institución de enseñanza superior en ambos años (año $1=91,30 \%$ y año $2=80,43 \%$ ), si bien al final del año 2 presenta más importancia para estos jóvenes trabajar (año $1=10,87 \%$ y año $2=21,74 \%$ ) o buscar un empleo (año $1=6,52 \%$ y año $2=19,56 \%$ ). Respecto a la expectativa de desempleo, los jóve- nes no piensan en esta opción como plan para el futuro, apuntando en cambio a estar trabajando de aquí a dentro de diez años en su área de interés (año $1=73,91 \%$ y año $2=71,74 \%$ ).

\section{Discusión}

En el estudio cualitativo de la voz de los jóvenes (estudio 1), los resultados subrayan la importancia de priorizar la voz de los jóvenes alrededor de temas relacionados con los gru- 
pos de iguales y con la sociedad. Algunos estudios han sostenido la importancia atribuida a los amigos y un cierto desencanto hacia la escuela (Matos, Gaspar, Cruz, \& Neves, 2013; Matos, Gaspar, Tomé, \& Cruz, 2012), en cambio el pensamiento crítico relacionado con la sociedad se ha mostrado en este estudio como un tema prioritario para los jóvenes. Esto sugiere la urgencia y necesidad de promover la relevancia del papel de los jóvenes en la sociedad, no sólo en la identificación de sus problemas y estrategias de la resolución, sino también en el proceso de toma de decisiones sobre políticas que directamente les afectan, potenciando un mayor y mejor entendimiento de los problemas de esta generación a través de su voz y experiencias (Kim, 2016). Además, esto viene a subrayar la pertinencia de aceptar a los jóvenes como un grupo social con su propio valor y de abandonar la visión de los jóvenes como problemas, aceptándolos en cambio como recursos importantes para la sociedad (Checkoway, 2011).

Por otra parte, en el estudio 2, la evaluación pre-post test del impacto del proyecto no mostró diferencias estadísticamente significativas en ninguna de las cinco dimensiones examinadas. Un resultado contradictorio, aunque en la línea de otra intervención anteriormente desarrollada en una comunidad portuguesa (Branquinho et al., 2018). En cambio, el estudio de la percepción del impacto sí revela algunas mejoras en el año 1 en cuestiones relacionadas con los sentimientos y competencias para la acción, competencias interpersonales y competencias para la resolución de problemas. En el año 2, se observó una evolución fa- vorable en asuntos referentes a sentimientos relacionados con la vida. Los estudios YPAR, han revelado mejoras significativas en algunas competencias posibles de ser integradas en las dimensiones creadas en este estudio, en particular, la capacidad de hablar en público, competencias de investigación, definición de objetivos y planificación de acciones (California Department of Public Health, 2011). De forma consistente con el estudio de Ozer y Douglas (2013), el presente trabajo también presentó un aumento del comportamiento participativo. Los resultados han señalado una mayor participación en actividades de liderazgo, en asociaciones de estudiantes y en la iglesia, así como en cargos de delegado o subdelegado de clase. La participación en actividades de voluntariado también es muy elevada, aunque presente una disminución del año 1 al año 2. En cambio, la participación en actividades de tutoría y apoyo de mentores adultos presenta un destacado incremento. Respecto a las perspectivas de futuro, la continuación de los estudios en la universidad o en una institución de enseñanza superior, disminuye en el año 2, cobrando importancia para los DT la idea de trabajar o ponerse a buscar un empleo. En su gran mayoría, los jóvenes tienen la expectativa de trabajar dentro de 10 años en su área vocacional.

A pesar de estos últimos resultados, posiblemente relacionados con la dificultad de los jóvenes para tratar con los desafíos sociales presentados durante el transcurso del proyecto, con el aumento de la concienciación de sus verdaderas competencias, o incluso del contexto en el que se desarrolló el proyec- 
to, se puede, de acuerdo con la Teoría de los Sistemas de Desarrollo propuesto por Lerner y Lerner (2013), creer que este programa ha contribuido al desarrollo del cuidado, carácter, conexión, confianza y competencia de los jóvenes participantes.

\section{Referencias}

Branquinho, C., \& Matos, M. G. (2018). The "Dream Teens" project: after a two-year participatory action-research program. Child Indicators Research, 12, 1243-1257.

Branquinho, C., Cruz, J., \& Matos, M. G. (2017). Dream Teens - a "voz" dos jovens na discussão de problemas da sua geração. Saúde e Desenvolvimento Humano, 5, 19-25. doi:10.18316/sdh.v5i3.3565

Branquinho, C., Fauvelet, C., Cruz, J., Santos, T., Gaspar, T., \& Matos, M. G. (2018). Dream Kids, dar voz às crianças: o futuro já começou, com autonomia e responsabilidade. Revista de Psicologia da Criança e do Adolescente, 8, 13-27.

Branquinho, C., Matos, M. G., \& Equipa Aventura Social / Dream Teens (2016). Dream Teens: Uma geração autónoma e socialmente participativa. En A. M. Pinto y R. Raimundo (Eds.), Avaliação e Promoção das Competências Socioemocionais em Portugal (pp. 421-440). Lisboa: Coisas de Ler.

California Department of Public Health (2011). Inspiring Youth, Growing Change. Recuperado de https://www.cdph.ca.gov/Programs/ CCDPHP/DCDIC/NEOPB/CDPH\%20Document\%20Library/PPPDS_InspiringYouthGC.pdf

Checkoway, B. (2011). Why is youth participation?. Children and Youth Services Review, 33, 340-345. doi: 10.1016/j. childyouth.2010.09.017

Frasquilho, D., Ozer, E., Ozer, E., Branquinho, C., Camacho, I., Reis, M., ... Matos, M. G. (2016). Adolescents-Led Participatory Project in Portugal in the Context of the Economic Recession. Health Promotion Practice, 19, 5159. doi:10.1177/1524839916660679

Hart, R. (1992). Children's Participation: From tokenism to citizenship. Florence: UNICEF.
Huebner, E. S. (1991). Initial development of the Students' Life Satisfaction Scale. School Psychology International, 12, 231-243. doi:10.1177/0143034391123010

Kia-Keating, M., Dowdy, E., Morgan, M. L., \& Noam, G. G. (2011). Protecting and promoting: an integrative conceptual model for healthy development of adolescents. Journal of Adolescence and Health, 48, 220-228. doi:10.1016/j.jadohealth.2010.08.006

Kim, J. (2016). Youth Involvement in Participatory Action Research (PAR): Challenges and barriers. Critical Social Work, 17, 38-53.

Lerner, R. M., \& Lerner, J. V. (2013). The Positive Development of Youth: Comprehensive Findings from the 4-h Study of Positive Youth Development. Connecticut, USA: National $4-\mathrm{H}$ Council.

Matos, M. G. (2015). Adolescents: safe navigation through unknown waters. Lisboa: Coisas de Ler.

Matos, M. G., Branquinho, C., Tomé, G., Camacho, C., Reis, M., Frasquilho, D., ... Equipa Aventura Social (2015). "Dream teens"Adolescentes autónomos, responsáveis e participantes, enfrentando a recessão em Portugal. Revista de Psicologia da Criança e do Adolescente, 6, 47-58.

Matos, M. G., Gaspar, T., Cruz, J., \& Neves, A. M. (2013). New highlights about worries, coping, and well-being during childhood and adolescence. Psychology Research, 3, 252260.

Matos, M. G., Gaspar, T., Tomé, G., \& Cruz, J. (2012). Worries and well-being during childhood and adolescence. Pakistan Journal of Clinical Psychology, 11, 3-14.

Muris, P. (2001). A brief questionnaire for measuring self-efficacy in youths. Journal of Psychopathology and Behavioral Assessment, 23, 145-149. doi:10.1023/A:1010961119608

Ozer, E., \& Douglas, L. (2013). The Impact of Participatory Research on Urban Teens: An Experimental Evaluation. American Journal of Community Psychology, 51, 66-75. doi:10.1007/s10464-012-9546-2.

Ozer, E. J., \& Schotland, M. (2011). Psychological empowerment among urban youth: Measure development and relation- 
ship to psychosocial functioning. Health Education \& Behavior, 38, 348-356. doi:10.1177/1090198110373734

Search Institute (2011). Profiles of Student Life: Attitudes and Behaviors. Recuperado de http://www.search-institute.org/sites/default/files/a/A\%26B\%202-page\%20sample\%202013.pdf

Shier, H. (2001). Pathways to participation: Openings, opportunities and obligations. Children \& Society, 15, 107-117. doi:10.1002/ chi.617.

Treseder, P. (1997). Empowering children and young people: Promoting involvement in decision-making. London: Save the Children.

UNICEF (2011). Every Child's Right to be Heard: a resource guide on the un committee on the rights of the child general comment no.12. Recuperado de https://www.unicef.org/ french/adolescence/files/Every_Childs_ Right_to_be_Heard.pdf
United Nations (2010). Youth Participation in Development: Summary guidelines for development partners. New York: United Nations.

World Health Organization (2014). Promoting mental health. Recuperado de http://www. who.int/mental_health/evidence/en/promoting_mhh.pdf

Zaff, J., Boyd, M., Li, Y., Lerner, J. V., \& Lerner, R. M. (2010). Active and engaged citizenship: multi-group and longitudinal factorial analysis of an integrated construct of civic engagement. Journal of Youth and Adolescence, 39, 736-750. doi:10.1007/s10964010-9541-6

Zeldin, S., Gauley, J., Krauss, S., Kornbluh, M., \& Collura, J. (2015). Youth-Adult Partnership and Youth Civic Development: Cross-National Analyses for Scholars and Field Professionals. Youth and Society, 49, 851-878. doi:10.1177/0044118X15595153

Agradecimientos: Agradecemos al equipo Aventura Social y los jóvenes del grupo Dream Teens su empeño en este proyecto. Financiación: Este proyecto fue financiado por las siguientes organizaciones: Fundación Calouste Gulbenkian, Sociedad Portuguesa de Psicología de la Salud, Facultad de Motricidad Humana de la Universidad de Lisboa, Programa ESCOLHAS, Instituto Portugués de Deporte y Juventud, y Universidad Lusíada de Lisboa.

Los autores de este trabajo declaran que no existe conflicto de intereses. 